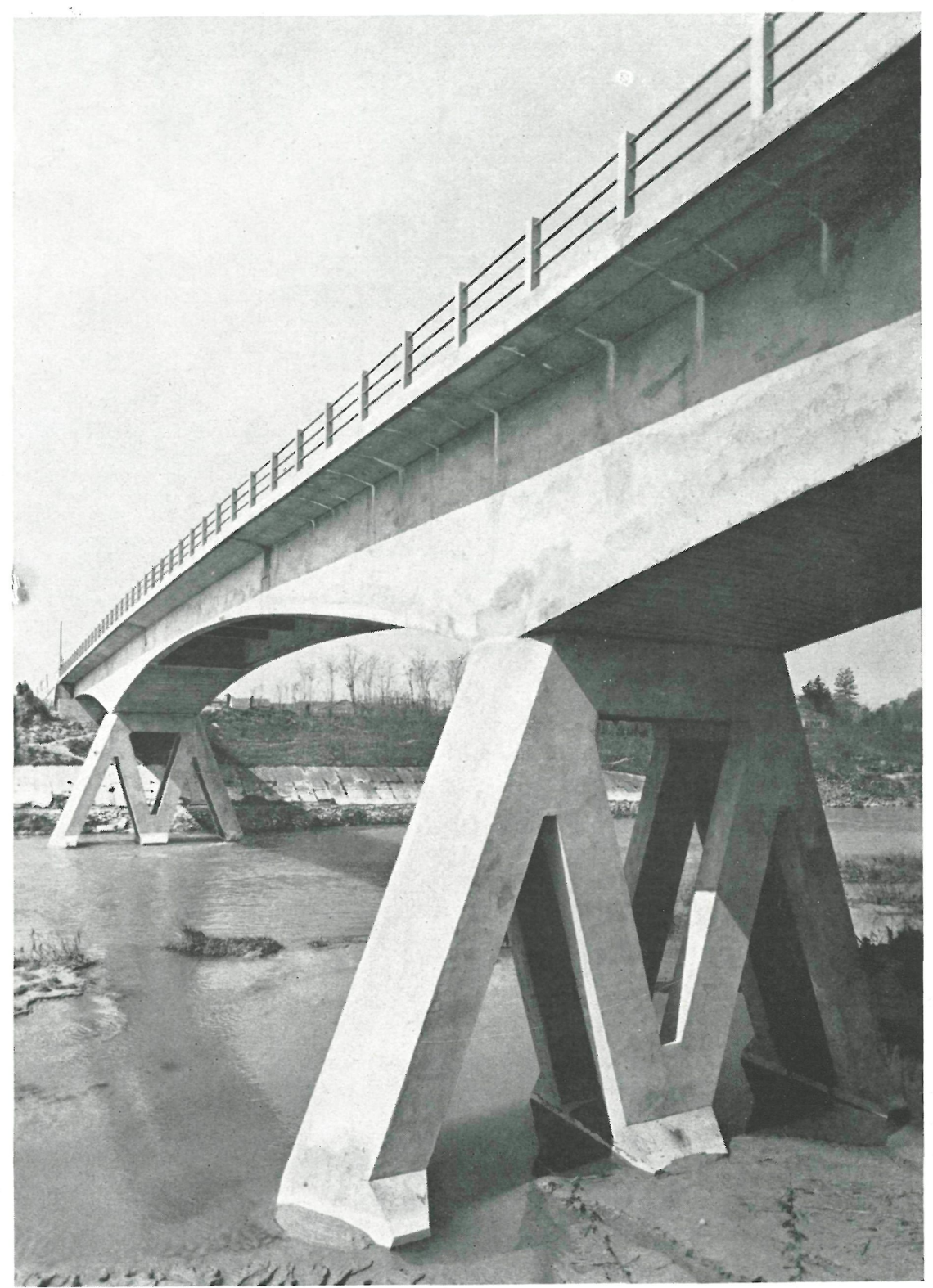

\title{
puente sobre el río Brenta
}

BELA SCHVARCZ, ingeniero

INSTITUTOTECNICODELACONSTRUCCION Y DEL CEMENTO 


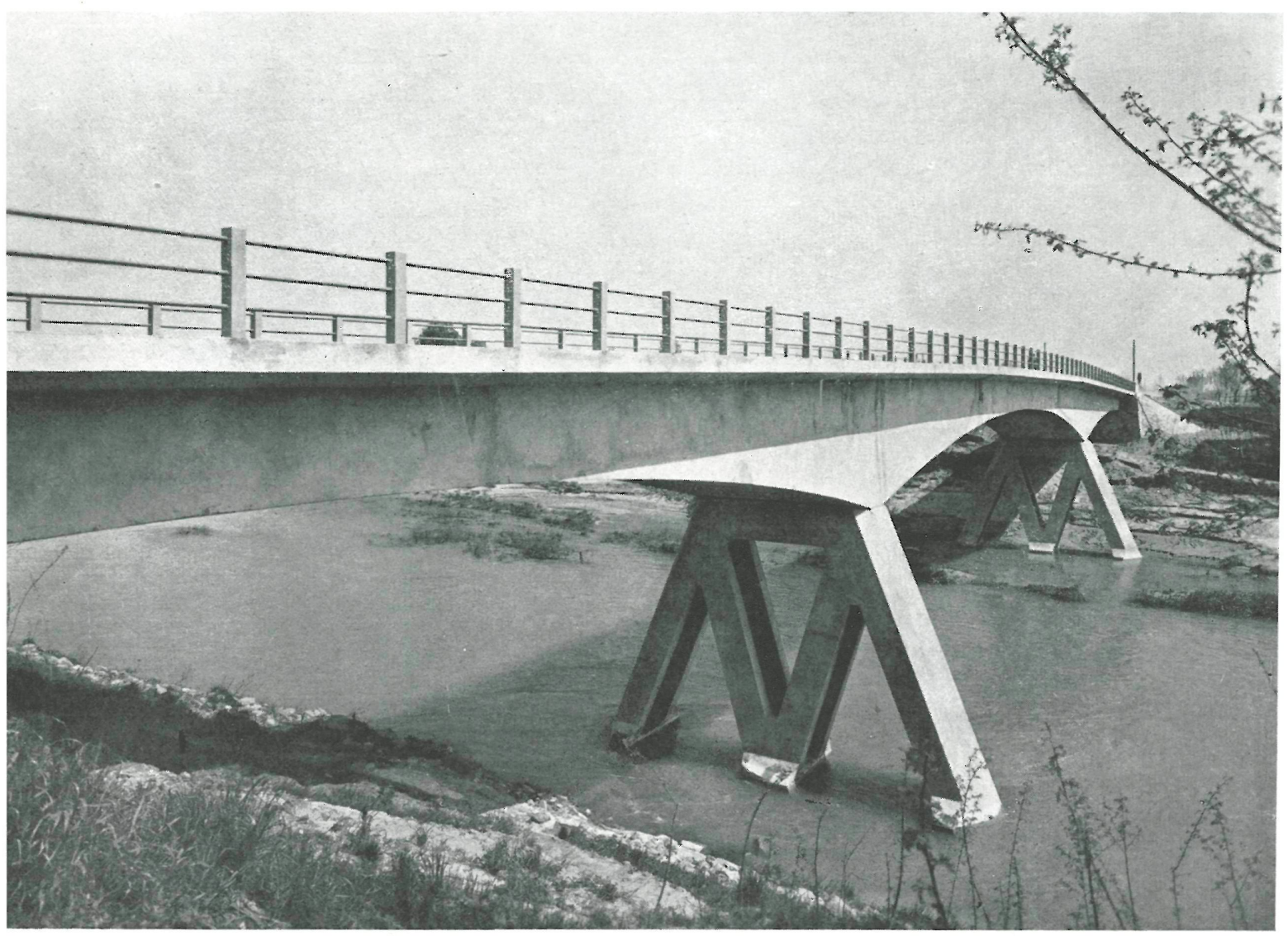

Aspecto general del puente mostrando los dos soportes intermedios en M y su armoniosa línea arquitectónica.

\section{Generalidades}

Para la unión por carretera de los poblados de Limena y Vigodarzere, de la provincia de Padua (Italia), separados por el río Brenta de Limena, se ha construído un puente de hormigón armado-proyectado por el autor-, de tres tramos, dos laterales de $26 \mathrm{~m}$ de luz cada uno y uno central de $50 \mathrm{~m}$, cuyo conjunto constituye un sistema Gerber con dos articulaciones situadas a $14 \mathrm{~m}$ de los apoyos intermedios y dentro del vano central del puente. La obra tiene $102 \mathrm{~m}$ de longitud total y un tablero subdividido en una calzada de $6 \mathrm{~m}$ de anchura y dos andenes de $1 \mathrm{~m}$ cada uno.

\section{Cimientos}

En principio se había proyectado la hinca de 80 pilotes, llevados a una profundidad de 12 a $14 \mathrm{~m}$ para aumentar la capacidad de carga de sustentación de un terreno no muy consistente y poder construir sobre ellos la solera de cimientos de los apoyos del puente. Pero unos sondeos previos pusieron de manifiesto que, a dicha profundidad, el terreno no presentaba suficiente seguridad para resistir las cargas previstas. clisposición de los soportes o pilas, ya que éstos debion aligerarse, en la medida de lo posible, lo que sucís una economía sop

Los pilotes, de hormigón, de unos $40 \mathrm{~cm}$ de diámetro, varían en profundidad y número, no ya en cada apoyo, sino en cada pila. La carga de sustentación admitida para cada uno de ellos es de 35 toneladas. Sobre las cabezas de estos elementos se ha formado un emparrillado o solera de base, sobre la que se ha construído el zócalo o dado del cuerpo emergente de la pila.

Las pilas o soportes intermedios son de hormigón armado, de $8,70 \mathrm{~m}$ de altura y muy aligerados por las razones anteriormente expuestas; pero, estructuralmente, estan constituidas por dos celosias en forma de $\mathbf{M}$, provistas de un cabezal en la parte superior y convenientemente espaciadas a 2,50 $\mathrm{m}$ entre los dos paramentos externos para darles gran estabilidad en el sentido longitudinal del puente.

En los soportes centrales, las reacciones resultantes son de 904 toneladas; y en los estribos, de unas 154

\section{Superestructura}

El tablero está soportado nor un sistema de dos vigas Gerber, apoyadas en los dos soportes centrales y estribos. Estas dos vigas, simplemente apoyadas, tienen un espesor de $50 \mathrm{~cm}$, a excepción de la parte de las pilas, donde el espesor pasa de 50 a $80 \mathrm{~cm}$ en una zona de transición de un metro de longitud; su altura varía de $1,60 \mathrm{~m}$ en estribos a 4,24 sobre las pilas, y, en el tramo central, de $1,70 \mathrm{~m}$ en la clave
a 2,25 en las articulaciones del trozo suspendido-de $22 \mathrm{~m}$ de longitud-por los dos voladizos de arranques. 


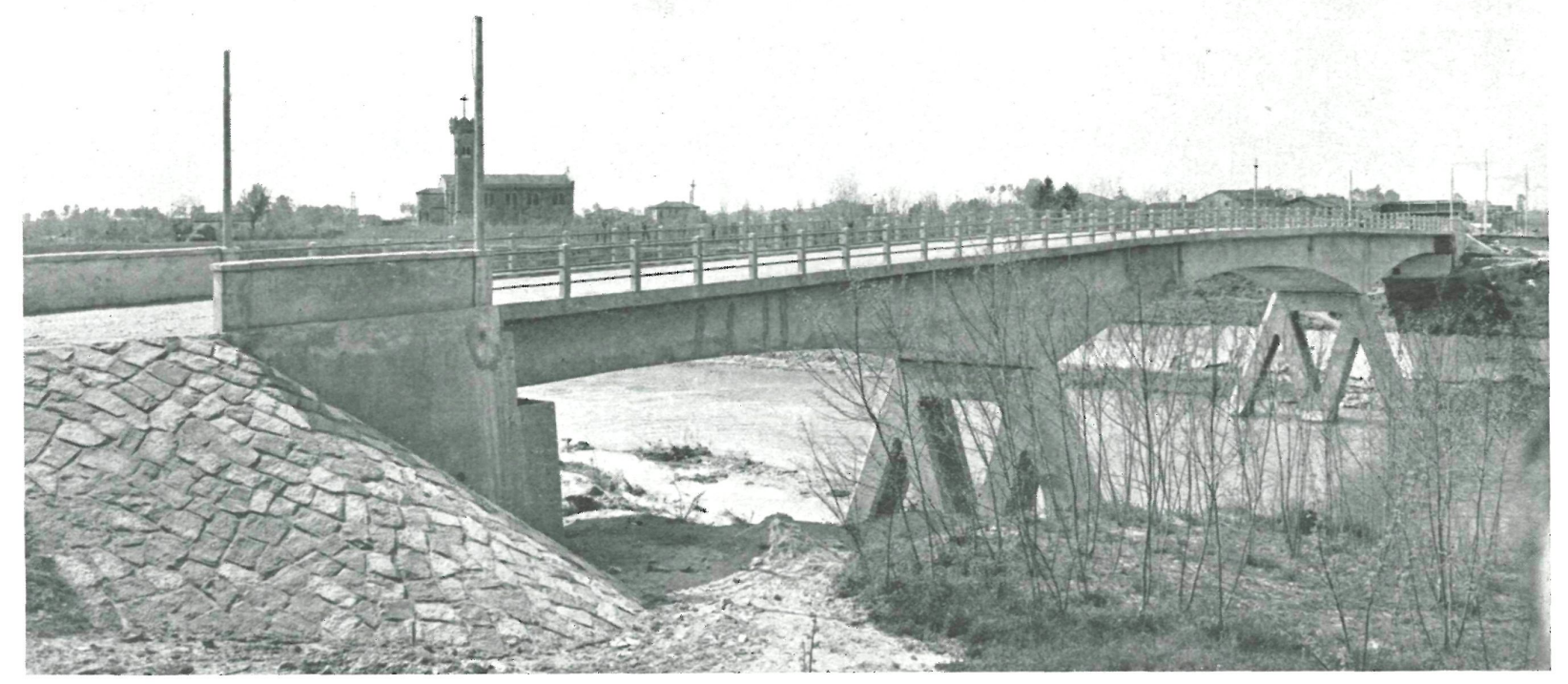

Los derrames del terraplén del acceso se han revestido con mampostería ordinaria para evitar la erosión.

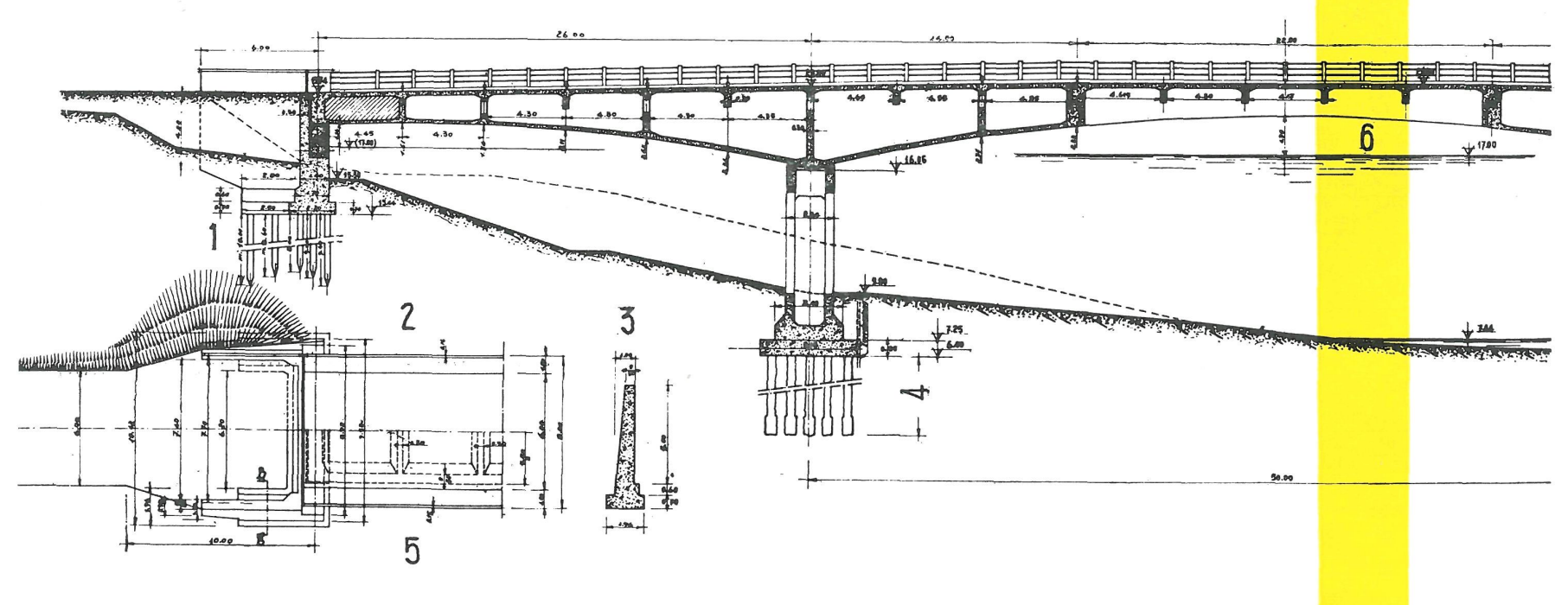

Obsérvense las dos articulaciones del trozo de cierre semi-alzado y planta Obsérvense las dos
del tramo central.

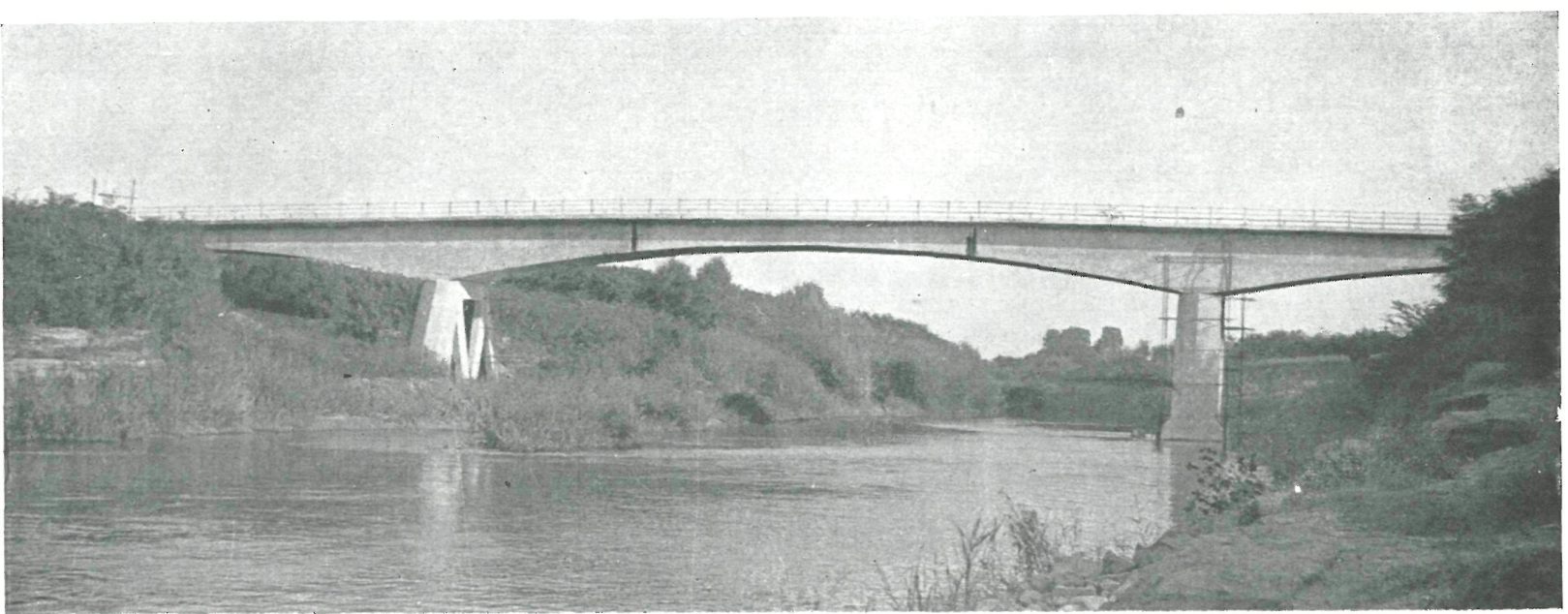




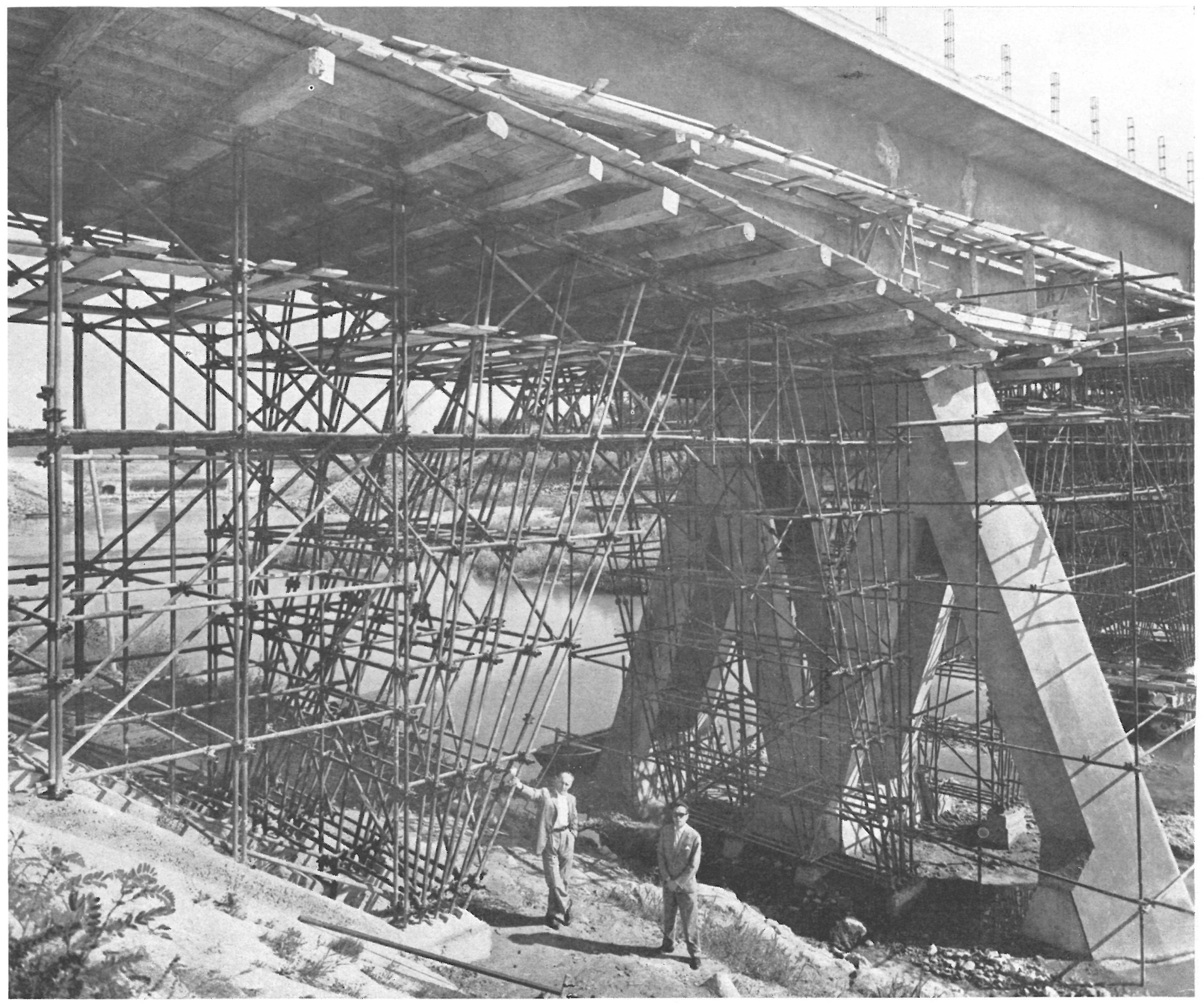

Entramado metálico tubular para soportar la cimbra.

Con objeto de dar al puente un aspecto arquitectónico satisfactorio, dada la altura de las pilas, así como lograr un sólido de casi resistencia uniforme, el intradós de los tramos de acceso es parabólico, con

Desde los estribos hasta la extremidad en voladizo del tramo central, las dos vigas, con sus diafragmas o tabiques transversales y losas de intradós variable de 15 a $25 \mathrm{~cm}$ de espesor, constituyen una viga cajón. A fin de obtener un equilibrio perfecto en las caras más desfavorables de cargas centrales, las dos cesario para la próximas a los estribos

Los estribos son de hormigón, apoyándose sobre ellos las vigas por medio de péndulos de hormigón, constituyendo rótulas que, en unión de los rodillos de la articulación del tramo central, permiten los movimientos originados por la retracción y cambios de temperatura.

La losa que constituye el extradós de la estructura, de $20 \mathrm{~cm}$ de espesor, recrecida con $5 \mathrm{~cm}$ de hormigon bituminoso, forma la calzada del puente, de $6 \mathrm{~m}$ de anchura, mientras que los andenes, de un metro de ancho, en voladizo respecto a las vigas, se han recrecido $14 \mathrm{~cm}$ con hormigón negro.

\section{Bases de cálculo}

Las tensiones que sirvieron para el proyecto del puente fueron de $60,4 \mathrm{~kg} / \mathrm{cm}^{2}$ para el hormigón del tablero trabajando a compresión, y $1.746 \mathrm{~kg} / \mathrm{cm}^{2}$ para el hierro de esta zona; en el tramo central, $66,8 \mathrm{~kg} / \mathrm{cm}^{2}$ para el hormigón y $1.745 \mathrm{~kg} / \mathrm{cm}^{2}$ para el hierro, y la de $76,8 \mathrm{~kg} / \mathrm{cm}^{2}$ para el hormigón y $1.795 \mathrm{~kg} / \mathrm{cm}^{2} \mathrm{par}$ el hierro en los tramos de acero. 


\section{secciones y detalles constructivos}

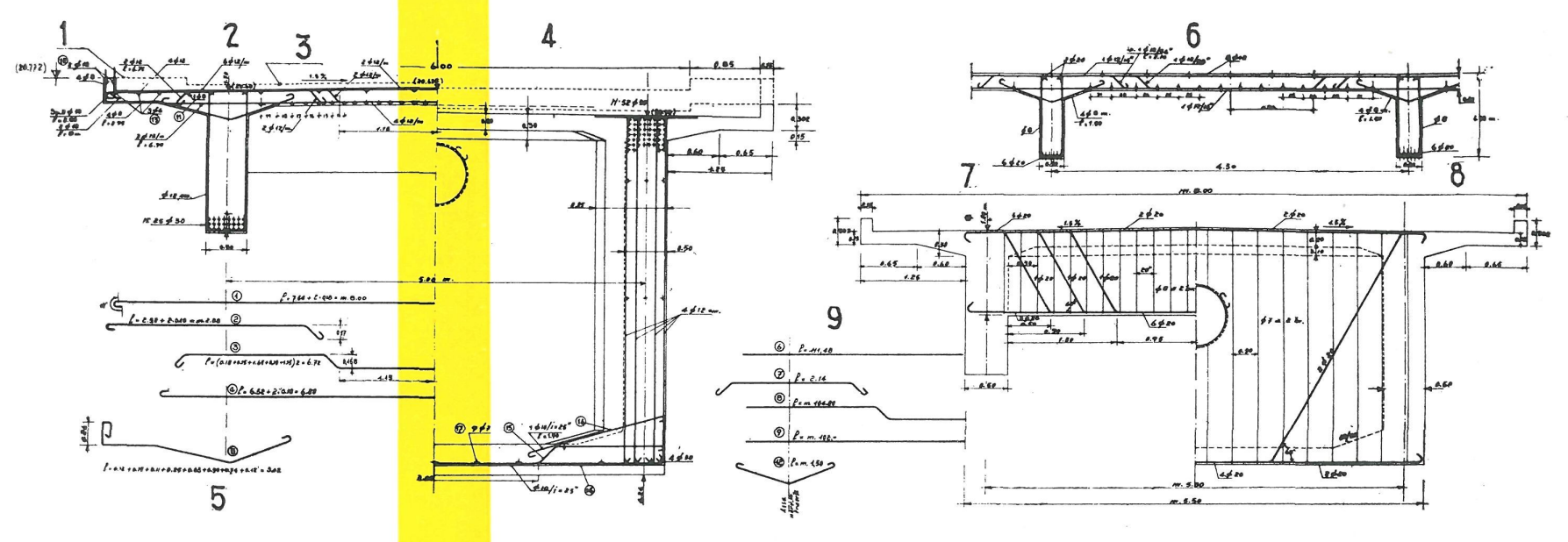

Para determinar estas tensiones se tuvieron en cuenta el estado de carga más desfavorable, una variación de temperatura de más o menos $30^{\circ} \mathrm{C}$, la retracción, acción del viento y efectos del empuje del agua.

\section{Métodos constructivos}

La obra se realizó utilizando los procedimientos actuales de construcción, sirviéndose de un entramado metálico-tubular para la formación de andamios.

Terminada la obra se sometió el puente a una serie de pruebas de cargas tipo, registrándose una flecha

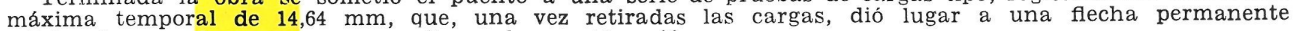
adicional por sobrecargas apenas digna de consideración.

Fotos: A. GIORDAN

Una fase de la ponstruccion de se ha terminae una de las dos partes en voladizo y se está hormigon and o la otra.

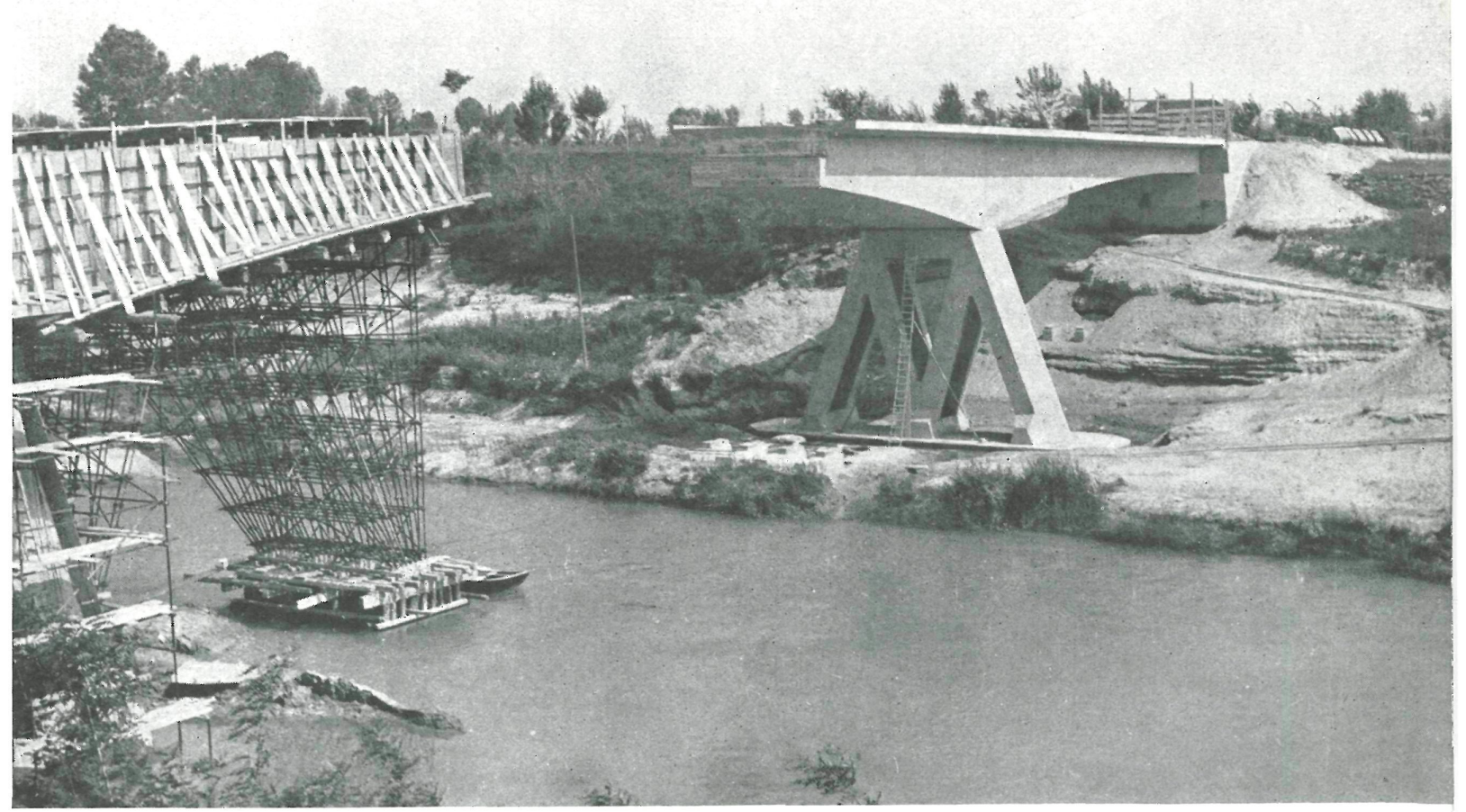

